TITLE:

\title{
Analytical expression of Capon spectrum for two uncorrelated signals using the inner product of mode vectors
}

\author{
$\operatorname{AUTHOR}(\mathrm{S}):$ \\ SAKAMOTO, Takuya; NISHIMURA, Koji
}

\section{CITATION:}

SAKAMOTO, Takuya ...[et al]. Analytical expression of Capon spectrum for two uncorrelated signals using the inner product of mode vectors. IEICE Transactions on Communications 2020, E103.B(4): 452-457

ISSUE DATE:

2020-04

URL:

http://hdl.handle.net/2433/252329

\section{RIGHT:}

(C) 2020 The Institute of Electronics, Information and Communication Engineers 


\title{
PAPER Analytical Expression of Capon Spectrum for Two Uncorrelated Signals Using the Inner Product of Mode Vectors
}

\author{
Takuya SAKAMOTO $^{\dagger, \dagger \uparrow a)}$ and Koji NISHIMURA ${ }^{\dagger \dagger \dagger}$, Members
}

SUMMARY An analytic expression of the Capon spectrum is derived for two uncorrelated incident signals. On the basis of this theoretical formulation, we discuss the effect of a factor arising from the inner product of mode vectors with respect to the incident angles, which compromises the resolution. We show numerical examples to demonstrate the effect that the inner product of mode vectors has on the shape of the Capon spectrum. key words: Capon method, antenna array, mode vectors

\section{Introduction}

The Capon method is well-known as a super-resolution algorithm for estimating directions of arrival using an antenna array. Although this method was proposed decades ago [1], its robustness and simple implementation have been of late driving its popularity and widespread use [2]-[9] including its application to sensing [10]-[14], antennas and propagation [15]-[19], and signal processing [20], [21]. Nonetheless, it is still extremely important to understand in an analytical way what determines the shape of the Capon spectrum. This is because the spectrum shape is affected by various factors when applying the Capon method to measured data. To assess the influence of each factor, an analytical expression is helpful and important to determine the cause of spectrum deformation that compromises the resolution.

An analytical spectrum obtained using the Capon method is derived for two uncorrelated incident signals and is compared with a simulated spectrum to evaluate the inner product of mode vectors (IPMV). Although Capon [1] derived such a spectrum for two incident waves, the contribution from the IPMV was not clear in his formula. The formula derived in this paper clearly explains the effect of the IPMV on the shape of the Capon spectrum.

Manuscript received April 23, 2019.

Manuscript revised August 6, 2019.

Manuscript publicized October 15, 2019.

${ }^{\dagger}$ The author is with the Department of Electrical Engineering, Graduate School of Engineering, Kyoto University, Kyoto-shi, 615-8510 Japan.

${ }^{\dagger}$ The author is also with Japan Science and Technology Agency, PRESTO, Kawaguchi-shi, 332-0012 Japan.

${ }^{\dagger \dagger}$ The author is with National Institute of Polar Research, Tachikawa-shi, 190-8518 Japan.

a) E-mail: sakamoto.takuya.8n@kyoto-u.ac.jp DOI: $10.1587 /$ transcom.2019EBP3096

\section{Beamformer and Capon Spectrum}

\subsection{System Model and Beamformer Method}

We assume a one-dimensional $N$-element linear antenna array and two uncorrelated signals (signals 1 and 2) with incident angles $\theta_{1}$ and $\theta_{2}$, satisfying $\theta_{1}<\theta_{2}$. The received signals are denoted $\boldsymbol{x}=\left[x_{1}, x_{2}, \cdots, x_{N}\right]^{\mathrm{T}}+\boldsymbol{n}$, where superscript $\mathrm{T}$ denotes the transpose operation and $\boldsymbol{n}$ is an independent and identically distributed $N$-dimensional white Gaussian noise vector. The correlation matrix $R$ is defined as $R=\mathrm{E}\left[\boldsymbol{x} \boldsymbol{x}^{\mathrm{H}}\right]$, where $\mathrm{E}[\cdot]$ denotes an expectation operator and superscript $\mathrm{H}$ denotes the conjugate transpose operation.

The spectrum of a beamformer (Fourier method) is expressed as $P_{\mathrm{F}}(\theta)=\boldsymbol{a}^{\mathrm{H}}(\theta) R \boldsymbol{a}(\theta)$, where $\boldsymbol{a}(\theta)$ is the mode vector defined by

$$
\boldsymbol{a}(\theta)=\left[1, \mathrm{e}^{\mathrm{j} k d \sin \theta}, \mathrm{e}^{\mathrm{j} 2 k d \sin \theta}, \cdots, \mathrm{e}^{\mathrm{j}(N-1) k d \sin \theta}\right]^{\mathrm{T}} .
$$

Here $k$ is the wavenumber and $d$ is the antenna spacing.

Given the two incident signals, $R$ is specifically expressed as

$$
R=a_{1}^{2} \boldsymbol{u}_{1} \boldsymbol{u}_{1}^{\mathrm{H}}+a_{2}^{2} \boldsymbol{u}_{2} \boldsymbol{u}_{2}^{\mathrm{H}}+\sigma^{2} I,
$$

where $a_{1}^{2}$ and $a_{2}^{2}$ denote the power of signals 1 and $2, \sigma^{2}$ is the power of the noise, $\boldsymbol{u}_{1}$ and $\boldsymbol{u}_{2}$ are the mode vectors for $\theta_{1}$ and $\theta_{2}$, respectively, and expressed as $\boldsymbol{u}_{1}=\boldsymbol{a}\left(\theta_{1}\right)$, and $\boldsymbol{u}_{2}=\boldsymbol{a}\left(\theta_{2}\right)$.

The beamformer spectrum then is expressed as

$$
P_{\mathrm{F}}(\theta)=N^{2} a_{1}^{2}\left|B_{1}(\theta)\right|^{2}+N^{2} a_{2}^{2}\left|B_{2}(\theta)\right|^{2}+\sigma^{2},
$$

where $B_{1}(\theta)=\boldsymbol{u}_{1}^{\mathrm{H}} \boldsymbol{a}(\theta) / N, B_{2}(\theta)=\boldsymbol{u}_{2}^{\mathrm{H}} \boldsymbol{a}(\theta) / N,\left|B_{1}(\theta)\right|^{2}=$ $\boldsymbol{a}^{\mathrm{H}}(\theta) \boldsymbol{u}_{1} \boldsymbol{u}_{1}^{\mathrm{H}} \boldsymbol{a}(\theta) / N^{2}$, and $\left|B_{2}(\theta)\right|^{2}=\boldsymbol{a}^{\mathrm{H}}(\theta) \boldsymbol{u}_{2} \boldsymbol{u}_{2}^{\mathrm{H}} \boldsymbol{a}(\theta) / N^{2}$. We note that the beams $B_{1}(\theta)$ and $B_{2}(\theta)$ have a dynamic range $0 \leq\left|B_{1}(\theta)\right|^{2},\left|B_{2}(\theta)\right|^{2} \leq 1$. They take maximum values of $\left|B_{1}\left(\theta_{1}\right)\right|^{2}=\left|B_{2}\left(\theta_{2}\right)\right|^{2}=1$ and minimum values of $\left|B_{1}(\theta)\right|^{2}=\left|B_{2}(\theta)\right|^{2}=0$ when $\theta$ satisfies $\boldsymbol{a}\left(\theta_{1}\right)^{\mathrm{H}} \boldsymbol{a}(\theta)=0$ and $\boldsymbol{a}\left(\theta_{2}\right)^{\mathrm{H}} \boldsymbol{a}(\theta)=0$, respectively.

\subsection{Derivation of the Theoretical Capon Spectrum}

The Capon spectrum is obtained by solving the following optimization problem

$$
\begin{gathered}
\boldsymbol{w}_{\mathrm{C}}(\theta)=\arg \min _{\boldsymbol{w}} \boldsymbol{w}^{\mathrm{H}} R \boldsymbol{w} \\
\text { subject to } \boldsymbol{w}^{\mathrm{H}} \boldsymbol{a}(\theta)=N,
\end{gathered}
$$


which can be solved using the method of Lagrange multipliers. With $\boldsymbol{w}_{\mathrm{C}}$ obtained as

$$
\boldsymbol{w}_{\mathrm{C}}(\theta)=\frac{N R^{-1} \boldsymbol{a}(\theta)}{\boldsymbol{a}^{\mathrm{H}}(\theta) R^{-1} \boldsymbol{a}(\theta)}
$$

then the Capon spectrum is finally given as

$$
P_{\mathrm{C}}(\theta)=\boldsymbol{w}_{\mathrm{C}}^{\mathrm{H}} R \boldsymbol{w}_{\mathrm{C}}=\frac{N^{2}}{\boldsymbol{a}^{\mathrm{H}}(\theta) R^{-1} \boldsymbol{a}(\theta)} .
$$

Equation (2) can be written as

$$
R=U T U^{\mathrm{H}}+\sigma^{2} I
$$

where $U=\left[\boldsymbol{u}_{1}, \boldsymbol{u}_{2}\right], T=\operatorname{diag}\left\{a_{1}^{2}, a_{2}^{2}\right\}$ and $I$ is a $N \times N$ identity matrix. Using the Woodbury matrix identity [22]-[24] (see Appendix), $R^{-1}$ is expressed as

$$
\begin{aligned}
R^{-1} & =\left(\sigma^{2} I+U T U^{\mathrm{H}}\right)^{-1} \\
& =\sigma^{-2} I-\sigma^{-4} U\left(T^{-1}+\sigma^{-2} U^{\mathrm{H}} U\right)^{-1} U^{\mathrm{H}},
\end{aligned}
$$

where $T^{-1}=\operatorname{diag}\left\{1 / a_{1}^{2}, 1 / a_{2}^{2}\right\}$. Thus,

$$
R^{-1}=\sigma^{-2} I-\sigma^{-4} U D^{-1} U^{\mathrm{H}},
$$

where

$$
D=\left[\begin{array}{cc}
1 / a_{1}^{2}+N / \sigma^{2} & N \rho / \sigma^{2} \\
N \rho^{*} / \sigma^{2} & 1 / a_{2}^{2}+N / \sigma^{2}
\end{array}\right] .
$$

We introduce the expression $\rho=\boldsymbol{u}_{1}^{\mathrm{H}} \boldsymbol{u}_{2} / N$, which reflects the similarity between the mode vectors $\boldsymbol{u}_{1}$ and $\boldsymbol{u}_{2}$. Next, we define the quantity IPMV because the similarity is calculated using the inner product of the vectors. The IPMV is a complex-valued constant written as

$$
\begin{aligned}
\rho & =\frac{1}{N} \sum_{l=0}^{N-1} \mathrm{e}^{j k d l\left(\sin \theta_{2}-\sin \theta_{1}\right)} \\
& =\frac{1}{N} \frac{\mathrm{e}^{j k d N\left(\sin \theta_{2}-\sin \theta_{1}\right)}-1}{\mathrm{e}^{j k d\left(\sin \theta_{2}-\sin \theta_{1}\right)}-1},
\end{aligned}
$$

which represents the inner product of mode vectors $\boldsymbol{u}_{1}$ and $\boldsymbol{u}_{2}$. We note that when the pair, $\theta_{1}$ and $\theta_{2}$, satisfies $k d N\left(\sin \theta_{2}-\sin \theta_{1}\right)=2 m \pi$ with $m$ integer, $\rho=0$ and the IPMV vanishes, and matrix $D$ becomes diagonal.

With det $D$ denoting the determinant of $D$,

$$
\operatorname{det} D \equiv\left(\frac{1}{a_{1}^{2}}+\frac{N}{\sigma^{2}}\right)\left(\frac{1}{a_{2}^{2}}+\frac{N}{\sigma^{2}}\right)-\frac{N^{2}|\rho|^{2}}{\sigma^{4}},
$$

then the inverse of the matrix $D$ is

$$
D^{-1}=\frac{1}{\operatorname{det} D}\left[\begin{array}{cc}
\frac{1}{a_{2}^{2}}+\frac{N}{\sigma^{2}} & -\frac{N \rho}{\sigma^{2}} \\
-\frac{N \rho^{*}}{\sigma^{2}} & \frac{1}{a_{1}^{2}}+\frac{N}{\sigma^{2}}
\end{array}\right],
$$

which leads to

$$
\begin{aligned}
U D^{-1} U^{\mathrm{H}}= & \frac{1}{\operatorname{det} D} \\
& \cdot\left\{\left(\frac{1}{a_{2}^{2}}+\frac{N}{\sigma^{2}}\right) \boldsymbol{u}_{1} \boldsymbol{u}_{1}^{\mathrm{H}}+\left(\frac{1}{a_{1}^{2}}+\frac{N}{\sigma^{2}}\right) \boldsymbol{u}_{2} \boldsymbol{u}_{2}^{\mathrm{H}}\right. \\
& \left.-\frac{N \rho}{\sigma^{2}} \boldsymbol{u}_{1} \boldsymbol{u}_{2}^{\mathrm{H}}-\frac{N \rho^{*}}{\sigma^{2}} \boldsymbol{u}_{2} \boldsymbol{u}_{1}^{\mathrm{H}}\right\} \\
= & \frac{\sigma^{2} / N}{\left(1+\gamma_{1}^{2}\right)\left(1+\gamma_{2}^{2}\right)-|\rho|^{2} \gamma_{1}^{2} \gamma_{2}^{2}} \\
& \cdot\left\{\gamma_{1}^{2}\left(1+\gamma_{2}^{2}\right) \boldsymbol{u}_{1} \boldsymbol{u}_{1}^{\mathrm{H}}+\gamma_{2}^{2}\left(1+\gamma_{1}^{2}\right) \boldsymbol{u}_{2} \boldsymbol{u}_{2}^{\mathrm{H}}\right. \\
& \left.-2 \gamma_{1}^{2} \gamma_{2}^{2} \operatorname{Re}\left[\rho \boldsymbol{u}_{1} \boldsymbol{u}_{2}^{\mathrm{H}}\right]\right\} \\
& =\frac{\sigma^{2}}{N}\left(c_{1} \boldsymbol{u}_{1} \boldsymbol{u}_{1}^{\mathrm{H}}+c_{2} \boldsymbol{u}_{2} \boldsymbol{u}_{2}^{\mathrm{H}}-c_{12} \operatorname{Re}\left\{\rho \boldsymbol{u}_{1} \boldsymbol{u}_{2}^{\mathrm{H}}\right\}\right),
\end{aligned}
$$

where $\gamma_{1}^{2}=N a_{1}^{2} / \sigma^{2}$ and $\gamma_{2}^{2}=N a_{2}^{2} / \sigma^{2}$ are the signal-tonoise ratios $(\mathrm{S} / \mathrm{N})$ for signals 1 and 2 that includes the gain of the array factor for $N$ elements. The coefficients $c_{1}, c_{2}$, and $c_{12}$ are defined as

$$
\begin{aligned}
& c_{1}=\frac{\gamma_{1}^{2}\left(1+\gamma_{2}^{2}\right)}{\left(1+\gamma_{1}^{2}\right)\left(1+\gamma_{2}^{2}\right)-|\rho|^{2} \gamma_{1}^{2} \gamma_{2}^{2}}, \\
& c_{2}=\frac{\gamma_{2}^{2}\left(1+\gamma_{1}^{2}\right)}{\left(1+\gamma_{1}^{2}\right)\left(1+\gamma_{2}^{2}\right)-|\rho|^{2} \gamma_{1}^{2} \gamma_{2}^{2}}
\end{aligned}
$$

and

$$
c_{12}=\frac{2 \gamma_{1}^{2} \gamma_{2}^{2}}{\left(1+\gamma_{1}^{2}\right)\left(1+\gamma_{2}^{2}\right)-|\rho|^{2} \gamma_{1}^{2} \gamma_{2}^{2}} .
$$

Finally, we obtain the inverse correlation matrix

$$
R^{-1}=\frac{1}{\sigma^{2}} I-\frac{1}{N \sigma^{2}}\left\{c_{1} \boldsymbol{u}_{1} \boldsymbol{u}_{1}^{\mathrm{H}}+c_{2} \boldsymbol{u}_{2} \boldsymbol{u}_{2}^{\mathrm{H}}-c_{12} \operatorname{Re}\left[\rho \boldsymbol{u}_{1} \boldsymbol{u}_{2}^{\mathrm{H}}\right]\right\} .
$$

Substituting this equation into Eq. (6), we obtain

$$
\begin{aligned}
P_{\mathrm{C}}(\theta) & =\frac{N^{3} \sigma^{2}}{\boldsymbol{a}^{\mathrm{H}}\left(N I-c_{1} \boldsymbol{u}_{1} \boldsymbol{u}_{1}^{\mathrm{H}}-c_{2} \boldsymbol{u}_{2} \boldsymbol{u}_{2}^{\mathrm{H}}+c_{12} \operatorname{Re}\left[\rho \boldsymbol{u}_{1} \boldsymbol{u}_{2}^{\mathrm{H}}\right]\right) \boldsymbol{a}} \\
& =\frac{N \sigma^{2}}{\left(1-c_{1}\left|B_{1}\right|^{2}-c_{2}\left|B_{2}\right|^{2}\right)+c_{12} \operatorname{Re}\left[\rho B_{1}^{*} B_{2}\right]} .
\end{aligned}
$$

Specifically, $B_{i}(\theta)(i=1,2)$ is written as

$$
\begin{aligned}
B_{i}(\theta) & =\frac{1}{N} \sum_{l=0}^{N-1} \mathrm{e}^{j k d l\left(\sin \theta-\sin \theta_{i}\right)}, \\
& =\frac{1}{N} \frac{\mathrm{e}^{j k d N\left(\sin \theta-\sin \theta_{i}\right)}-1}{\mathrm{e}^{j k d\left(\sin \theta-\sin \theta_{i}\right)}-1} .
\end{aligned}
$$

By substituting Eq. (20) to Eq. (19), the Capon spectrum for two incident waves can be written explicitly.

Note that the simple expression in Eq. (19) was derived using the Woodbury matrix identity, whereas Capon [1] instead used the Sherman-Morrison formula [25] expressed 
as $\left(A+\boldsymbol{u} \boldsymbol{v}^{\mathrm{T}}\right)^{-1}=A^{-1}-A^{-1} \boldsymbol{u} \boldsymbol{v}^{\mathrm{T}} A^{-1} /\left(1+\boldsymbol{v}^{\mathrm{T}} A^{-1} \boldsymbol{u}\right)$, which is a special case of the Woodbury matrix identity [22]. Cox [26] applied the Sherman-Morrison formula twice and obtained a similar equation. However, the theoretical spectrum (19) is not found in their papers [1], [26]. Equation (19) gives a clear perspective on the spectral shape because it explicitly shows the IPMV contribution to the resultant spectrum.

\subsection{Capon Spectrum in Special Cases}

In a special case where there is only a single incident signal, i.e., $a_{1}^{2} \neq 0, a_{2}^{2}=0$ (thus $\gamma_{1}^{2} \neq 0$ and $\gamma_{2}^{2}=0$ ), $c_{2}$ and $c_{12}$ both vanish, and thus the Capon spectrum is written as

$$
P_{\mathrm{C}}(\theta)=N \sigma^{2} \frac{1+\gamma_{1}^{2}}{1+\gamma_{1}^{2}\left(1-\left|B_{1}(\theta)\right|^{2}\right)} .
$$

In this case, $P_{\mathrm{C}}(\theta)$ has a dynamic range

$$
N \sigma^{2} \leq P_{\mathrm{C}}(\theta) \leq N \sigma^{2}\left(1+\gamma_{1}^{2}\right) .
$$

We define the beamwidth $W_{\mathrm{BF}}$ of $\left|B_{1}(\theta)\right|^{2}$ as $W_{\mathrm{BF}}=\mid \theta_{3 \mathrm{~dB}}^{(2)}-$ $\theta_{3 \mathrm{~dB}}^{(1)} \mid$, where $\left|B_{1}\left(\theta_{3 \mathrm{~dB}}^{(1)}\right)\right|^{2}=\left|B_{1}\left(\theta_{3 \mathrm{~dB}}^{(2)}\right)\right|^{2}=1 / 2$. Substituting $\theta=\theta_{3 \mathrm{~dB}}^{(i)}(i=1,2)$ into Eq. $(21)$, we obtain

$$
P_{\mathrm{C}}\left(\theta_{3 \mathrm{~dB}}^{(i)}\right)=N \sigma^{2} \frac{1+\gamma_{1}^{2}}{1+\gamma_{1}^{2} / 2}
$$

Dividing Eq. (23) by the maximum value of $P_{\mathrm{C}}(\theta)$ gives

$$
P_{\mathrm{C}}\left(\theta_{3 \mathrm{~dB}}^{(i)}\right) / \max _{\theta} P_{\mathrm{C}}(\theta)=\frac{1}{1+\gamma_{1}^{2} / 2} .
$$

From Eq. (24), we observe that the Capon spectrum $P_{\mathrm{C}}(\theta)$ has a sharper peak than the beamformer spectrum $\left|B_{1}(\theta)\right|^{2}$, which corresponds to the condition $1 /\left(1+\gamma_{1}^{2} / 2\right)<1 / 2$ if $\gamma_{1}^{2}>2$ corresponding to $\mathrm{S} / \mathrm{N}>3 \mathrm{~dB}$.

In another special case, if the mode vectors for $\theta_{1}$ and $\theta_{2}$ are orthogonal, i.e. IPMV vanishes $(\rho=0)$, and if the signals have the same power $a_{1}^{2}=a_{2}^{2}$, the Capon spectrum simplifies,

$$
P_{\mathrm{C}}(\theta)=N \sigma^{2} \frac{1+\gamma_{1}^{2}}{1+\gamma_{1}^{2}\left(1-\left|B_{1}(\theta)\right|^{2}-\left|B_{2}(\theta)\right|^{2}\right)},
$$

which seems a straightforward extension of the single-signal case in Eq. (21). The spectrum also has two peaks at $\theta_{1}$ and $\theta_{2}$, both peaks having the same width as the single-signal case. The dynamic range of $P_{C}(\theta)$ is also the same as the single-signal case as in Eq. (22). We note that as long as IPMV $\rho$ is sufficiently small, the resolution of the Capon method can be deduced simply from the beam width of the Capon spectrum discussed above. In contrast, when $\rho$ is nonzero, the term $c_{12} \operatorname{Re}\left[\rho B_{1}^{*} B_{2}\right]$ affects the shape of the spectrum unlike single-signal cases.

\section{Numerical Examples}

We next compare the analytical Capon spectrum in Eq. (19)

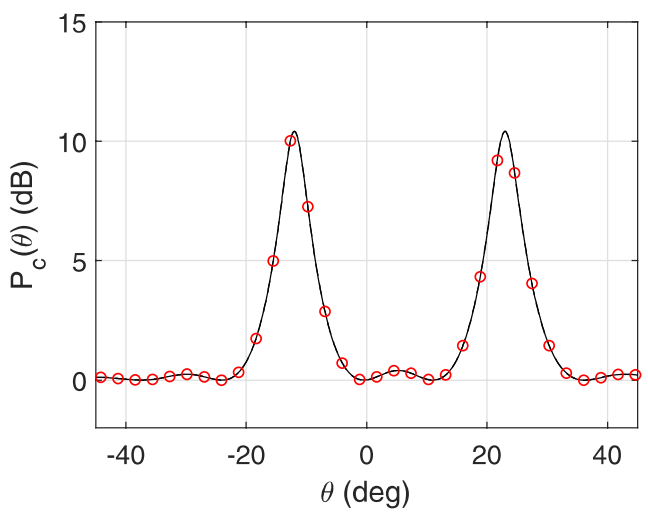

Fig. 1 The theoretical (black solid line), approximated with $\rho=0$ (black dashed line), and the simulated (red circles) Capon spectra for $N=10$, $\theta_{1}=-12^{\circ}, \theta_{2}=23^{\circ}$, and $\gamma_{1}^{2}=\gamma_{2}^{2}=10$.

and the simulated spectrum. We assume hereafter halfwavelength antenna spacings $d=\lambda / 2$. For a 10-element array $(N=10), \theta_{1}=-12^{\circ}, \theta_{2}=23^{\circ}, \sigma^{2}=0.1$ and $\gamma_{1}^{2}=\gamma_{2}^{2}=10$ corresponding to $S / N=10 \mathrm{~dB}$, the theoretical (black solid line) and simulated (red circles) Capon spectra are compared (Fig. 1), for which the simulated spectrum was calculated using Eq. (6) and $R$ given by Eq. (2), which corresponds to an infinite number of snapshots, completely uncorrelated signals, and white uncorrelated noise components. The result indicates good agreement between the two spectra. In this case, IPMV was $|\rho|=2.6 \times 10^{-3}$. Next, we set $\rho=0$ in the analytical spectrum expressions to evaluate the contribution of $\rho$ to the spectrum shape. The approximated spectrum with $\rho=0$ is drawn as a black dashed line in Fig. 1, which almost completely overlaps the theoretical spectrum (black solid line), indicating that the effect of $\rho$ is not significant compared with the spectrum floor ( 0 $\mathrm{dB})$. The root mean square (RMS) errors of the strict and approximated spectra were $3.5 \times 10^{-15}$ and $2.4 \times 10^{-3}$, which indicate that the inner product of the mode vectors does not significantly affect the Capon spectrum in this case. We remark that the RMS error $\varepsilon$ was calculated from

$$
\varepsilon=\sqrt{\frac{1}{\pi} \int_{-\pi / 2}^{\pi / 2}\left|P_{\mathrm{C}}(\theta)-P_{\operatorname{sim}}(\theta)\right|^{2} \mathrm{~d} \theta}
$$

where $P_{\mathrm{C}}(\theta)$ and $P_{\text {sim }}(\theta)$ are the analytical and simulated Capon spectra, respectively.

We present another example of a 10-element array $(N=10), \theta_{1}=2^{\circ}, \theta_{2}=19^{\circ}, \sigma^{2}=0.1$ and $\gamma_{1}^{2}=\gamma_{2}^{2}=10$ corresponding to $S / N=10 \mathrm{~dB}$. The analytical, approximated and simulated spectra are shown as a black solid line, black dashed line, and red circles in Fig. 2. It is observed that the approximated spectrum in the figure overestimates the peaks, giving erroneously sharper beams. In this case, the modulus of the IPMV $|\rho|=0.22$, a value which is larger than that of the previous case. The RMS errors of the strict and approximated $(\rho=0)$ spectra were $3.7 \times 10^{-15}$ and 1.4. In this case, the IPMV has a significant influence on the Capon spectrum unlike the previous case. 


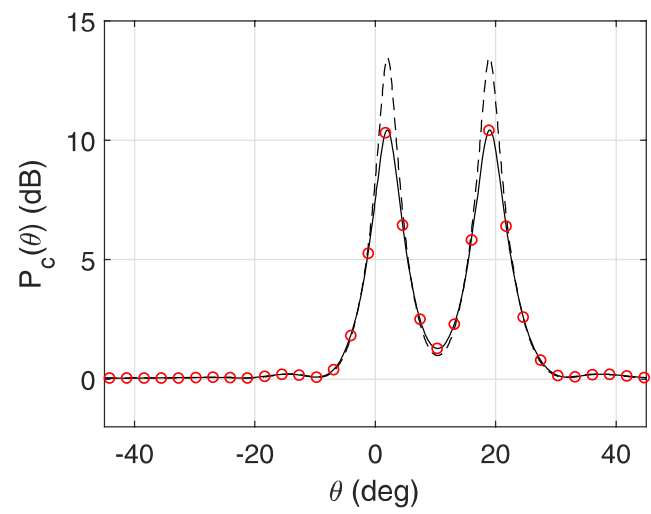

Fig. 2 The theoretical (black solid line), approximated $(\rho=0)$ (black dashed line), and simulated (red circles) Capon spectra for $N=10, \theta_{1}=2^{\circ}$, $\theta_{2}=19^{\circ}$, and $\gamma_{1}^{2}=\gamma_{2}^{2}=10$.

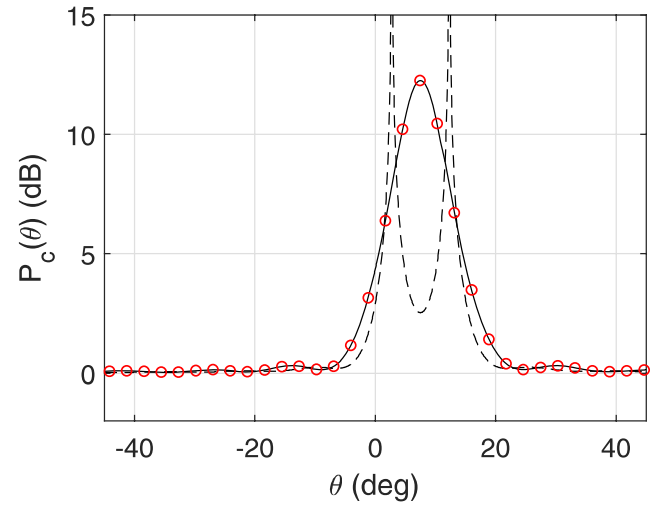

Fig. 3 The theoretical (black line), approximated with $\rho=0$ (black dashed line), and simulated (red circles) Capon spectra for $N=10, \theta_{1}=5^{\circ}$, $\theta_{2}=12^{\circ}$ and $\gamma_{1}^{2}=\gamma_{2}^{2}=10$.

Figure 3 shows the theoretical, approximated, and simulated spectra for a 10-element array $(N=10), \theta_{1}=5^{\circ}$, $\theta_{2}=12^{\circ}, \sigma^{2}=0.1$ and $\gamma_{1}^{2}=\gamma_{2}^{2}=10$ corresponding to $S / N=10 \mathrm{~dB}$. In this case, the IPMV $|\rho|=0.50$ was larger than those in the previous cases and the RMS errors of the strict and approximated spectra were $8.4 \times 10^{-15}$ and 13.0. Similar to the previous case, nonzero IPMV gives erroneously sharp peaks of the dashed line in Fig. 3, whereas the two peaks are barely resolved in the theoretical and simulated spectra, which indicates that the effect of IPMV is critical in determining the resolution of the Capon method.

Figure 4 shows color-coded values for the modulus squared of the IPMV $|\rho|^{2}$ for various settings of the pair $\theta_{1}$ and $\theta_{2}$ for $N=10$; three settings, $\left(\theta_{1}, \theta_{2}\right)=\left(-12^{\circ}, 23^{\circ}\right)$, $\left(2^{\circ}, 19^{\circ}\right)$, and $\left(5^{\circ}, 12^{\circ}\right)$ are marked by black, red and yellow crosses. We see relatively small and large IPMVs for $\left(\theta_{1}, \theta_{2}\right)=\left(-12^{\circ}, 23^{\circ}\right)$ and $\left(5^{\circ}, 12^{\circ}\right)$, respectively; they explain the discrepancy seen in the approximated spectra and also different RMS errors. The IPMV has a quasi-periodic feature arising from the cyclic mode vectors. For comparison, the IPMV $|\rho|^{2}$ for $N=4$ is shown in Fig. 5. We see a longer period in the variation of the IPMV.

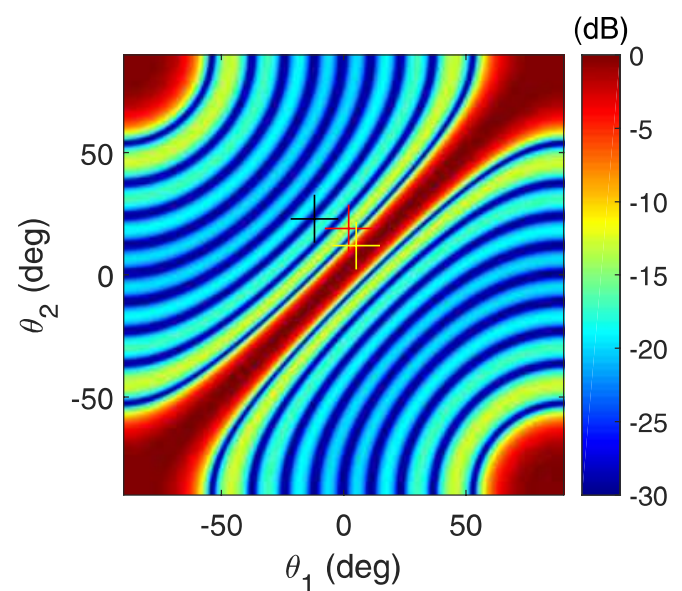

Fig. 4 The modulus squared of IPMV $|\rho|^{2}$ for various incident-angle paris $\theta_{1}$ and $\theta_{2}(N=10)$. Cross symbols indicate the incident angles for Figs. 1 (black), 2 (red) and 3 (yellow).

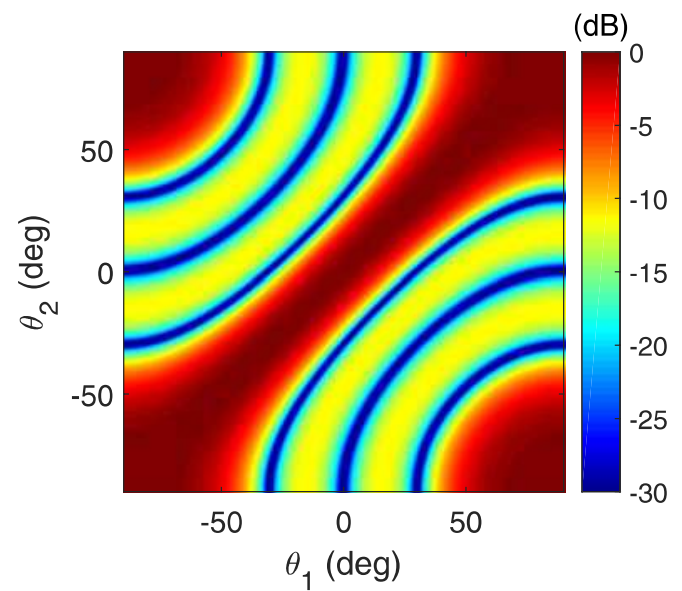

Fig. 5 The modulus squared of the IPMV $|\rho|^{2}$ for various incident-angle pairs $\theta_{1}$ and $\theta_{2}(N=4)$.

\section{Conclusion}

We have derived analytically a Capon spectrum for two uncorrelated incident signals. The derived formula includes an IPMV corresponding to the incident angles. Its effect was demonstrated through numerical examples and compared with simulated Capon spectra. The value of the IPMV was evaluated for different incident-angle pairs. Although we analyzed a case with two incident signals, a similar component of the mode vectors would have a significant effect in the general case with more than two signals. The derived formula helps to provide a clear and intuitive understanding of the shape of the Capon spectrum.

\section{Acknowledgments}

This study was supported in part by JSPS KAKENHI Grants (No. 19H02155, 15K18077 and 15KK0243), JST PRESTO Grant Number JPMJPR1873, and JST COI Grant Number 
JPMJCE1307. We would like to thank Prof. Toru Sato of the Institute for Liberal Arts and Sciences, Kyoto University, for providing valuable advice on this research.

\section{References}

[1] J. Capon, "High-resolution frequency-wavenumber spectrum analysis," Proc. IEEE, vol.57, no.8, pp.1408-1418, Aug. 1969

[2] P. Stoica, Z. Wang, and J. Li, "Robust Capon beamforming," IEEE Signal Process. Lett., vol.10, no.6, pp.172-175, June 2003

[3] J. Li, P. Stoica, and Z. Wang, "On robust Capon beamforming and diagonal loading," IEEE Trans. Signal Process., vol.51, no.7, pp. 1702-1715, July 2003.

[4] A. Hassanien, S. Shahbazpanahi, and A.B. Gershman, "A generalized Capon estimator for localization of multiple spread sources," IEEE Trans. Signal Process., vol.52, no.1, pp.280-283, Jan. 2004.

[5] J. Li, P. Stoica, and Z. Wang, "Doubly constrained robust Capon beamformer," IEEE Trans. Signal Process., vol.52, no.9, pp.24072423, Sept. 2004.

[6] J. Benesty, J. Chen, and Y. Huang, "A generalized MVDR spectrum,” IEEE Signal Process. Lett., vol.12, no.12, pp.827-830, Dec. 2005

[7] A. Jakobsson, S.R. Alty, and J. Benesty, "Estimating and timeupdating the 2-D coherence spectrum," IEEE Trans. Signal Process., vol.55, no.5, pp.2350-2354, May 2007.

[8] G.-O. Glentis, "A fast algorithm for APES and Capon spectral estimation,” IEEE Trans. Signal Process., vol.56, no.9, pp.4207-4220, May 2008.

[9] N.R. Butt and A. Jakobsson, "Coherence spectrum estimation from nonuniformly sampled sequences," IEEE Signal Process. Lett., vol.17, no.4, pp.339-342, April 2010.

[10] P. Lopez-Dekker and J.J. Mallorqui, "Capon- and APES-based SAR processing: performance and practical considerations," IEEE Trans. Geosci. Remote Sens., vol.48, no.5, pp.2388-2402, May 2010.

[11] S.D. Somasundaram, A. Jakobsson, and N.H. Parsons, "Robust and automatic data-adaptive beamforming for multidimensional arrays," IEEE Trans. Geosci. Remote Sens., vol.50, no.11, pp.4642-4656, Nov. 2012.

[12] H. Bi, B. Zhang, W. Hong, and S. Zhou, "Matrix-completion-based airborne tomographic SAR inversion under missing data," IEEE Geosci. Remote Sens. Lett., vol.12, no.11, pp.2346-2350, Nov. 2015.

[13] H. Park, V. González-Gambau, and A. Camps, "High angular resolution RFI localization in synthetic aperture interferometric radiometers using direction-of-arrival estimation," IEEE Geosci. Remote Sens. Lett., vol.12, no.1, pp.102-106, Jan. 2015.

[14] M. Pardini and K. Papathanassiou, "On the estimation of ground and volume polarimetric covariances in forest scenarios with SAR tomography," IEEE Geosci. Remote Sens. Lett., vol.14, no.10, pp.1860-1864, Oct. 2017.

[15] A. Elnashar, S.M. Elnoubi, and H.A. El-Mikati, "Further study on robust adaptive beamforming with optimum diagonal loading," IEEE Trans. Antennas Propag., vol.54, no.12, pp.3647-3658, Dec. 2006

[16] J.-S. Hu and M.-T. Lee, "Norm-constrained Capon beamforming using multirank signal models with Kalman filter implementation," IEEE Trans. Antennas Propag., vol.62, no.9, pp.4574-4583, Sept. 2014

[17] S. Kikuchi, H. Tsuji, and A. Sano, "Autocalibration algorithm for robust Capon beamforming," IEEE Antennas Wireless Propag. Lett., vol.5, pp.251-255, June 2006

[18] Y. Liu and Q. Wan, "Sidelobe suppression for robust Capon beamforming with mainlobe-to-sidelobe power ratio maximization," IEEE Antennas Wireless Propag. Lett., vol.11, pp.1218-1221, 2012.

[19] M.D. Hossain and A.S. Mohan, "Eigenspace time-reversal robust
Capon beamforming for target localization in continuous random media," IEEE Antennas Wireless Propag. Lett., vol.16, pp.16051608, Jan. 2017

[20] Y. Ke, C. Zheng, R. Peng, and X. Li, "Robust adaptive beamforming using noise reduction preprocessing-based fully automatic diagonal loading and steering vector estimation," IEEE Access, vol.5 pp.12974-12987, July 2017.

[21] D. Igambi, X. Yang, and B. Jalal, "Robust adaptive beamforming based on desired signal power reduction and output power of spatial matched filter," IEEE Access, vol.6, pp.50217-50228, Sept. 2018

[22] M.A. Woodbury, Inverting modified matrices, Memorandum report 42, Statistical Research Group, Princeton University, Princeton, NJ 1950 .

[23] X. Xu, "Generalization of the Sherman-Morrison-Woodbury formula involving the Schur complement," Appl. Math Comput., vol.309, pp.183-191, Sept. 2017.

[24] W.W. Hager, "Updating the inverse of a matrix," SIAM Rev., vol.31, no.2, pp.221-239, June 1989.

[25] J. Sherman and W.J. Morrison, "Adjustment of an inverse matrix corresponding to a change in one element of a given matrix," Ann. Math. Statist., vol.21, no.1, pp.124-127, 1950.

[26] H. Cox, "Resolving power and sensitivity to mismatch of optimum array processors," J. Acoust. Soc. Am., vol.54, no.3, pp.771-785 Sept. 1973.

\section{Appendix:}

The Woodbury matrix identity [22] is found to be

$$
(A+U C V)^{-1}=A^{-1}-A^{-1} U\left(C^{-1}+V A^{-1} U\right)^{-1} V A^{-1} .
$$

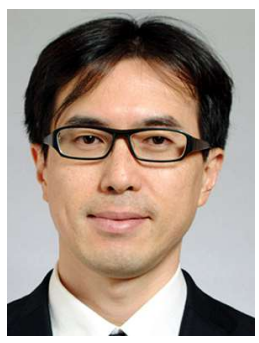

Takuya Sakamoto received a B.E. degree in electrical and electronic engineering from Kyoto University, Kyoto, Japan, in 2000 and M.I. and $\mathrm{Ph}$.D. degrees in communications and computer engineering from the Graduate School of Informatics, Kyoto University, in 2002 and 2005, respectively. From 2006 through 2015, he was an Assistant Professor at the Graduate School of Informatics, Kyoto University. From 2011 through 2013, he was also a Visiting Researcher at Delft University of Technology, Delft, the Netherlands. From 2015 through 2019, he was an Associate Professor at the Graduate School of Engineering, University of Hyogo, Himeji, Japan, and a Part-time Researcher at the Graduate School of Informatics, Kyoto University. In 2017, he was also a Visiting Scholar at the University of Hawaii at Manoa, Honolulu, HI, USA. Since 2018, he has also been PRESTO researcher of the Japan Science and Technology Agency, Kawaguchi, Japan. Currently, he is an Associate Professor at the Graduate School of Engineering, Kyoto University. His current research interests lie in radar signal processing, inverse scattering, electromagnetic imaging and applied systems theory. In 2016, Dr. Sakamoto received Masao Horiba Award. In 2017, he was invited as a semi-plenary speaker of the European Conference on Antennas and Propagation (EuCAP) in Paris, France. He is a Senior Member of IEEE 


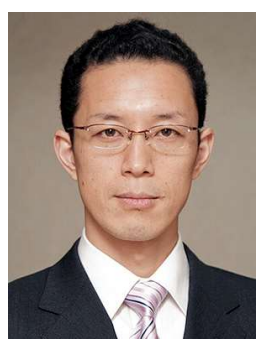

Koji Nishimura received his B.E. degree from Ritsumeikan University, Japan, in 1999, and his M.I. and Ph.D. degrees from Kyoto University, Japan, in 2001 and 2006, respectively. From 2001 to 2003, he worked for Sony Corporation. Since 2007 , he has been with the National Institute of Polar Research, Tokyo, Japan, where he is now a project associate professor. His major research interests are radar signal processing, multi-channel signal processing, remote sensing for the atmosphere, and satellite communications. In 2014, Dr. Nishimura received The Prize for Science and Technology (in The Commendation for Science and Technology by the Minister of Education, Culture, Sports, Science and Technology) from the Japanese government. 